\title{
Introducing Clinical Trials Highlights
}

\author{
Kevin McFarthing, $\mathrm{PhD}$
}

\begin{abstract}
Welcome to a Journal of Parkinson's Disease (JPD) rarity - an editorial written by a Person with Parkinson's (PwP). I'm a biochemist and even though I don't actively work in science now, a scientist is always a scientist; and a PwP never stops being a PwP - yet. I also compile the Hope List, a comprehensive database of Parkinson's therapies in development, from research phase through to Phase 3.
\end{abstract}

I'm delighted to be the joint editor, together with Prof. Tanya Simuni of Northwestern University, of the new Clinical Trials Highlights section of JPD. As a PwP, I applaud the editors for inviting me to be a section editor, recognising the pivotal role played by $\mathrm{PwP}$ in trials as well as acknowledging the work done to advocate for faster, more targeted and effective trial outcomes.

The objective of Clinical Trial Highlights is to raise awareness of the clinical trial landscape in Parkinson's, promoting discussion and progress in the conduct and outcome of studies. It will be a resource centre for academics, medics, industry and PwP, particularly those wanting to participate in clinical trials. We will review ongoing and completed trials in specific areas for each edition. Other resources will be available online, including links to clinicaltrials.gov; Fox Trial Finder; pdtrialtracker.info; and the Hope List. Phase 3 studies need particular attention as they are the closest to market, so each edition of Clinical Trial Highlights will have a Phase 3 Focus, selecting one study for analysis.

In short, we want Clinical Trial Highlights to be the primary destination for anybody with a vested interest in the success of trials in Parkinson's. So why are we doing this?

The pace and progress of Parkinson's research is unparalleled, with ground breaking results reported on an almost monthly basis. We have probably learned more about Parkinson's in the last twenty years than in the two hundred since James Parkinson described his eponymous disease. Indeed, the current era of Parkinson's research may be termed a "golden age". Yet the grey-haired and wrinkled levodopa is still the "gold standard" of treatment, with no drugs proven to slow or stop the progress of disease. How do we explain this strange dichotomy?

It isn't for lack of effort. It is despite the massive dedication and commitment of both researchers and trial participants, as well as the enormous financial investment needed to conduct clinical trials.

The bald truth is that we face fundamental problems in translating research into clinical practice. Parkinson's remains a complicated condition with unclear etiology yet inexorable progression. It is heterogeneous, multi-target, variable both inter- and intra-patient and difficult to diagnose and prognose. It's a disease verging on a syndrome.

Neurologists face daily challenges trying to tailor the best treatment to each individual sat opposite. Clinical trial designers must overcome the heterogeneity to produce results that they hope are not only statistically sound, but relevant to as many patients as possible. All too often we produce a statistical and clinical average, unable to identify and subsequently match novel treatments to the ideal recipients.

Taking a strict scientific approach would mean abandoning clinical trials until we have a full understanding of what goes wrong, enabling a link between genotype and phenotype; we have clear, validated and accurate biomarkers; and we can predict progression at an individual level. But I, for one PwP, can't wait. Somehow, we need to blend science and clinical trial 
design with urgency in a cocktail that solves the clinical conundrum. We want Clinical Trial Highlights to contribute to this vision.

The first edition focuses on $\alpha$-synuclein targeted therapeutics, primarily immunotherapies, describing studies that are currently in the clinical phase. This is a very exciting area, with a mix of approaches aimed at altering the course of disease progression by preventing the aggregation of $\alpha$-synuclein into oligomers and fibrils; and increasing clearance of those already formed.
The Phase 3 Focus this month is the repurposing of isradipine, a calcium channel blocker. This important trial is due to report in 2019, so this edition's article will describe the design and set the scene for the impending results.

We're excited about Clinical Trial Highlights and welcome comments and feedback on future editions. Please help us to make a difference to the outcome of clinical trials in Parkinson's, looking forward to a time when clinical progress matches the performance in the lab. 